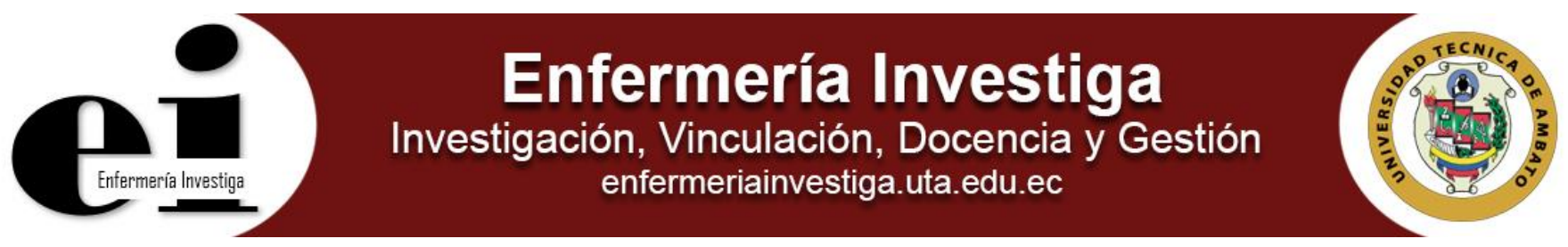

DOI: http://dx.doi.org/10.29033/ei.v3sup1.2018.02

Artículo de revisión

\title{
Consideraciones sobre el impacto de la morbilidad y mortalidad neonatal y pediátrica en la salud pública ecuatoriana
}

Considerations on the impact of neonatal and pediatric morbidity and mortality on the ecuadorian public health

Jessica Maritza Martínez Cantos¹, Mónica Vinueza²

${ }^{1}$ Médico Especialista en Pediatría - Hospital General Docente de Calderón - Quito - Ecuador

${ }^{2}$ Médico Especialista en Pediatría - Hospital General Docente de Calderón - Quito - Ecuador

Martínez CJM, Vinueza M. Consideraciones sobre el impacto de la morbilidad y mortalidad neonatal y pediátrica en la salud pública ecuatoriana. Enferm Inv (Ambato). 2018; 3(Sup.1): 9-14

2477-9172 / 2550-6692 Derechos Reservados ( 2018 Universidad Técnica de Ambato, Carrera de Enfermería. Este es un artículo de acceso abierto distribuido bajo los términos de la Licencia Creative Commons, que permite uso ilimitado, distribución y reproducción en cualquier medio, siempre que la obra original es debidamente citada.

\section{Historia:}

Recibido: 27 noviembre 2017

Revisado: 13 diciembre 2017

Aceptado: 30 diciembre 2017

Palabras Claves: Morbimortalidad neonatal; morbimortalidad pediátrica; salud pública ecuatoriana

Keywords: Neonatal morbidity and mortality; pediatric morbidity and mortality; ecuadorian public healt

\section{Resumen}

La morbimortalidad materno fetal incide sobre el sistema sanitario de forma relevante, si recordamos que constituye un indicador de desarrollo social y humano. Derivado de la preocupación que despierta el estado de la problemática en el ámbito regional, el presente artículo pretende sistematizar en la literatura algunos elementos relacionados con el impacto de la morbi-mortalidad neonatal y pediátrica en la salud pública ecuatoriana y reflexionar sobre ello. Se concluye que se hace evidente las inequidades que aún persisten en la sociedad ecuatoriana, en particular, el acceso a servicios de salud, aunque se identificaron pautas para su trasformación se requiere de un programa de seguimiento que ayude a la reducción de las mismas. En este sentido, la atención básica en el nivel primario de atención desempeña un importante papel para la promoción del cuidado a los neonatos de riesgo y a los infantes.
Abstract

Maternal and fetal morbidity and mortality affects the health system in a relevant way, if we remember that it constitutes an indicator of social and human development. Derived from the concern raised by the state of the problem at the regional level, this article aims to systematize in the literature some elements related to the impact of neonatal and pediatric morbidity and mortality in Ecuadorian public health and to reflect upon it. It is concluded that the inequalities that still persist in Ecuadorian society are evident, in particular, access to health services, although guidelines for their transformation were identified, a follow-up program is needed to help reduce them. In this sense, basic care at the primary care level plays an important role in the promotion of care to high-risk infants and infants. 


\section{Introducción}

La mortalidad infantil es uno de los indicadores de desarrollo más importante para cualquier país y está relacionado con el desarrollo del embarazo, el parto y el puerperio; además ofrece información preliminar sobre el estado o calidad del sistema sanitario en cuestión. La Organización Mundial de la Salud (OMS) reporta que cada año en el mundo nacen alrededor de 15 millones de niños y niñas, cerca del $10 \%$ no llega a los cinco años pues fallece de diarreas, neumonías, entre otras y ocho millones fallecen antes del año de vida. ${ }^{1}$

La mayoría de estas muertes se producen en países del tercer mundo de África, Asia, América del Norte y América latina, y todas están relacionadas con el déficit o dificultades en los cuidados médicos básicos, lo que habla de un sistema de salud deficiente. ${ }^{2}$ En tal sentido, los países han reconocido la necesidad de reforzar el acceso a los servicios de salud, darle mayor prioridad a la atención profesional durante el embarazo, parto y el puerperio; así mismo han mostrado acuerdo en que la mortalidad materno infantil es una emergencia global por lo que hay que trabajar sobre factores económicos, nutricionales, educacionales y fortalecer los vínculos con redes de apoyo locales y regionales.

Si bien la mortalidad infantil es un indicador de desarrollo también está relacionado con la implementación de planes y políticas sanitarias que contribuyan a reducir la muerte de la madre y su hijo alrededor del nacimiento y posterior al mismo, la morbilidad en el grupo de niños en situación de riesgo también se vuelve relevante, ya que puede llevar a la muerte debido a la mayor vulnerabilidad de este grupo, principalmente en los casos de infecciones respiratorias agudas y afecciones perinatales. ${ }^{3}$ Se reporta en otras investigaciones que entre las principales causas de morbilidad neonatal e infantil se encuentran los problemas respiratorios al momento del nacimiento, el bajo peso al nacer, el nacimiento prematuro, las sepsis neonatales, las malformaciones congénitas, entre otras. ${ }^{4-6}$ Las principales causas de muerte entre los menores de cinco años son la neumonía y los problemas de malnutrición, según la OMS.

La morbimortalidad neonatal o infantil se puede prevenir, se afirma que una atención prenatal donde se controlen factores de riesgo que complican el curso del embarazo sería lo ideal y se recomienda que las mujeres reciban entre cinco y trece controles durante todo el embarazo. Afirma que cada año nacen niños prematuros y las complicaciones relacionadas con la prematuridad son la principal causa de defunción en los niños menores de 5 años; así en el año 2013 provocaron cerca de un millón de muertes y las tres cuartas partes de esas muertes podrían evitarse con intervenciones eficaces e incluso sin necesidad de recurrir a servicios de cuidados intensivos. ${ }^{7}$ Luego del nacimiento, se debe procurar que el niño reciba lactancia materna exclusiva durante los primeros seis meses de vida ${ }^{8}$, lo cual resulta eficaz en la prevención de enfermedades y garantiza una mejor alimentación y desarrollo del niño sobre todo si vive en países del tercer mundo. ${ }^{9}$

Recomiendan también el cumplimiento de esquemas de inmunizaciones en mujeres en edades fértiles y embarazadas, se ha probado que disminuye los riesgos de transmisión de enfermedades. Se sugiere el tratamiento de cualquier enfermedad crónica, infecciones localizadas durante la gestación el periparto o el puerperio sobre la base de los protocolos prescrito para ello10,11

\section{La morbimortalidad neonatal y pediátrica y su influencia en la salud pública latinoamericana}

La morbimortalidad materno fetal incide sobre el sistema sanitario de forma relevante, si recordamos que constituye un indicador de desarrollo social y humano para los países. Los estados y gobiernos a través de sus carteras de salud han identificado causas de muertes evitables que con la implementación de acciones o intervenciones sanitarias de diagnóstico, tratamiento, promoción y atención a la salud reducen los riesgos a la mínima expresión para la madre o el niño.

En Brasil se han organizado agendas que buscan organizar la asistencia sanitaria a través de líneas de cuidados que ayude a dar continuidad a los cuidados entre los tres niveles de atención de forma planificada y ordenada para superar la desarticulación de manera ágil y oportuna en cada nivel de atención (primaria, secundaria y terciaria), con referencia y contra-referencia responsable, hasta la recuperación completa del individuo. ${ }^{12}$

Una de las líneas de cuidado es precisamente la atención integral de la salud del niño, en la misma se prioriza la atención al recién nacido $(\mathrm{RN})$ de riesgo. La finalidad de la línea es la reducción de la mortalidad infantil, la atención humanizada y cualificada a la gestante y al $\mathrm{RN}$ e incentivo de la lactancia materna. ${ }^{13}$ Con el nacimiento del niño se organiza la atención de seguimiento al recién nacido por la unidad de salud, en la que se realizan visitas domiciliarias diarias durante la primera semana de vida, se recogen los datos del niño para organizar la tarjeta o cartilla de control del menor, se priorizan los cuidados, se realiza acompañamiento a la madre y al niño, se identifican riesgos y se preparara a la madre para el cuidado de su hijo.

Se ha señalado que alrededor de un $72.7 \%$ de las muertes neonatales ocurridas en países latinoamericanos están asociadas a BPN. De los recién nacidos con bajo peso que sobreviven, se calcula que entre 13 y $24 \%$ padecen trastornos neurológicos y entre 6 y $13 \%$, déficit intelectual, lo que repercute en la adaptación social y calidad de 
vida. El bajo peso al nacer es el índice predictivo más importante de mortalidad infantil y el factor fundamental asociado con los más de 5 millones de defunciones neonatales que ocurren cada año en el mundo. ${ }^{14}$

Las causas más frecuentes de reingreso precoz de neonatos son la ictericia, los problemas con el amamantamiento y la deshidratación/desnutrición. La causa más frecuente de reingreso tardío son las infecciones respiratorias. El riesgo es mayor en los de menor tiempo de gestación, en los de menor peso y en los varones. ${ }^{15}$

En Chile, un estudio reveló que los niños que nacen prematuramente sufren complicaciones a corto, mediano y largo plazo, y que se ven afectados por diferentes complicaciones y morbilidades asociadas al nacimiento con efectos que afectan su niñez y la etapa adulta con alto costo físico, psicológico y económico. ${ }^{16}$

En los Estados Unidos se realizó un reporte de los costos que para el sistema sanitario tenía implícito el nacimiento de un niño prematuro. Se estimó en el año 2005 los gastos médicos, educativos y pérdida de productividad asociada al parto prematuro, las que fueron superiores a US\$ 26,2 mil millones. ${ }^{13}$ Los promedios de los costos médicos del primer año de atención hospitalaria y ambulatoria, eran 10 veces mayor para prematuros (US\$32.325) que para neonato a término (US\$3.325). La estancia media fue 9 veces más larga para un neonato prematuro (13 días), que uno a término ( 1,5 días). ${ }^{17}$

En Perú, las estrategias empleadas para reducir la morbimortalidad neonatal e infantil se dirigió la atención a la institucionalización del parto en los sectores de la sierra, la selva, el rural y el área urbana. Así mismo, se mejoró el acceso a los servicios de salud reforzando la atención con seguro de salud lo que implica resolver la capacidad resolutiva para afrontar la demanda de atención, se mejoró el programa de vacunación donde se aplica vacunas a la madre durante el período prenatal y luego al niño posterior al nacimiento y se práctica el amamantamiento temprano el mismo que ayuda a prevenir enfermedades pues restablece el vínculo madre-hijo. ${ }^{17}$

En Colombia, se reporta la problemática de vulnerabilidad y alto riesgo de neonatos hijos de madres adolescentes. Al respecto se afirma que la maternidad en las etapas tempranas de la vida es un problema grave para la salud pública, trae consigo mayor riesgo para la salud reproductiva y propicia un alto riesgo para la salud de la adolescente y su hijo, aumentado la morbilidad, la mortalidad, también la exclusión social por perpetuación de la pobreza. ${ }^{18}$ Se asume que la adolescente embarazada tiene mayores riesgos de salud materna y neonatal, mayor morbilidad, mortalidad materna y neonatal, dificultades para la formación escolar y laboral, problemas de convivencia con sus padres y su pareja, se embaraza más frecuentemente entre adolescentes pobres, tienen mayor probabilidad de ser madres solteras y enfrentar la ausencia e irresponsabilidad de los padres y no hace ejercicio de derechos y es víctima de la inequidad de género.

Las estadísticas relacionadas con la morbilidad del neonato suelen afectarse cuando la población de nacidos prematuros y nacidos a término con bajo peso son susceptibles de presentar problemas en los primeros días de vida relacionados fundamentalmente con riesgos de hipotermia e hipertermia, hipoglucemia, hiperbilirrubinemia, problemas en la alimentación, deshidratación y desnutrición. Razones por las que el neonato necesita atención y cuidados especiales. A pesar de los cuidados, el riesgo de reingreso precoz y tardío es mayor en el neonato, realidad que enfrenta el equipo de salud y la madre del niño. ${ }^{18}$

Derivado de la preocupación que despierta el estado de la problemática en el ámbito regional, el presente artículo pretende sistematizar en la literatura algunos elementos relacionados con el impacto de la morbi-mortalidad neonatal y pediátrica en la salud pública ecuatoriana y reflexionar sobre ello.

\section{Desarrollo}

En la bibliografía estudiada, se identificó que las características socioeconómicas, tales como la escolaridad del cabeza de familia, el número de habitaciones de la vivienda y la localización del domicilio actúan como factores de riesgo asociados negativamente a la mortalidad neonatal precoz y a la baja renta familiar. ${ }^{19}$

Se reconoce que los óbitos neonatales pueden evitarse si se logra el pleno acceso a los servicios de salud, pues su evitabilidad está más relacionada con las condiciones de la atención prenatal y el parto, que con la existencia de equipamiento técnico sofisticado. ${ }^{13}$ De esta manera los recién nacidos que viven en hogares con malas condiciones de habitabilidad hacen al RN más vulnerable a los problemas de salud. Así mismo se reconoce al lugar de residencia como factor de riesgo relacionado con la morbimortalidad neonatal, pues el lugar o localización de la vivienda pudiera afectar el acceso a los servicios de salud, pues si se considera la estructura de atención, localización y calidad de los servicios, el acceso a los bienes y servicios de salud es restringido. ${ }^{20}$

Como se ha detallado anteriormente, se requiere abordar esta problemática en nuestro contexto, ya que la morbimortalidad neonatal es un problema de salud pública en el Ecuador debido a que sus causas son diversas y la mayoría prevenible desde el nivel primario de atención. Al mismo tiempo la reducción de este indicador constituye uno de los objetivos de desarrollo del milenio planteado por la OMS: reducir la mortalidad infantil en dos terceras partes desde 1990. Para ello se deben generar investigaciones que profundicen en las causas de la muerte y se desarrollen protocolos de actuación que favorezcan la calidad en la atención. 
Por otro lado, para reducir la morbimortalidad neonatal es necesario la puesta en práctica de acciones durante el embarazo que capaciten adecuadamente a la gestante. ${ }^{12}$ Se precisa de una infraestructura sanitaria con el mejor equipamiento técnico y talento humano suficiente que contribuya a identificar tempranamente los factores de riesgos, reducir las complicaciones y mejorar las condiciones en las que se atiende al neonato o el niño.

Se reconoce que el mayor riesgo de mortalidad materna, por ende, morbimortalidad infantil corresponde a las adolescentes de menos de 15 años. Se afirma que son las complicaciones del embarazo y el parto las causas de muerte principales de las adolescentes en la mayoría de los países en desarrollo por lo que la atención a este grupo vulnerable debe ser continua y sistemática si consideramos que la mayoría de estas complicaciones aparecen durante la gestación y pueden ser prevenibles o tratables; aunque se debe reconocer que pueden existir otras que se agravan con la gestación. ${ }^{4-8}$

La mayoría de las complicaciones durante el embarazo, parto y puerperio ocurren en mujeres de bajos recursos económicos, bajo nivel de instrucción y que no han recibido buena atención de salud. Por ello, se considera que las mujeres pobres de zonas lejanas son las que tienen menos probabilidades de recibir una atención sanitaria adecuada lo que significa que muchos de esos partos no son asistidos por un médico o una partera, situación que puede poner en peligro la vida de la madre y/o del niño. 4-8

También se registran otros factores que se convierten en limitaciones para el acceso a los servicios de salud de las mujeres de bajos ingresos y sus hijos, entre ellos se encuentran la pobreza, las largas distancias entre su lugar de vivienda y los centros de salud; la falta de información; la inexistencia de servicios básicos adecuados como el agua y el alcantarillado y algunas prácticas culturales que no contribuyen al mejoramiento de las condiciones de vida de la familia, por ende, tampoco favorece una infancia saludable. Son estas razones las que pudieran hacer pertinente la realización de estudios donde se profundice en las causas y condiciones que favorecen la morbimortalidad del neonato y del niño. .-10 $^{-10}$

\section{Impacto de la morbilidad neonatal y pediátrica en la salud pública ecuatoriana}

La OMS diseñó la Estrategia de Atención Primaria de Salud donde se proponen atención y cuidados esenciales a los niños todos ellos sustentados científicamente y al alcance de todas las familias, apelando a la plena participación ciudadana y a un costo módico para la sociedad y el país. Las alternativas se implementan en cada etapa de la vida del niño de forma responsable. ${ }^{21}$

Siguiendo esta lógica, el gobierno ecuatoriano, bajo la estrategia del Plan Nacional del Buen Vivir, dedica a la Salud a través de sus lineamientos un lugar de especial. Por ello se concibió e implementa el Plan Nacional de Salud por medio del cual se ha reducido considerablemente las muertes maternas, neonatales e infantiles en comparación con períodos precedentes a la existencia de estos programas o estrategias dirigidas a mejorar la atención de la madre y el niño.

Resulta necesario comentar que las enfermedades que hoy afectan a los neonatos y la niñez se dan en familias de bajos o escasos recursos económicos, donde se identifican limitaciones para el acceso a servicios básicos (agua segura, electricidad, alcantarillado) y por ende, dichas familias habitan en deficientes condiciones de salubridad que afecta su calidad de vida y propicia la aparición de enfermedades. A esto se le añade el bajo nivel de escolaridad de las madres y padres que inciden directamente sobre las condiciones de vida de la familia, repercute negativamente en la salud y se revierte en gastos para el sistema sanitario. ${ }^{22}$

En reportes realizados por la OMS, se plantea que en Ecuador entre 1993 y el 2000 murieron 93 mujeres por cada 100000 nacidos vivos, y para el 2006 las cifras habían disminuido a 53 muertes por cada 100000 . En tanto, se reportaba que la mortalidad materna global mundial era de 400 mujeres por cada 100000 nacidos vivos, cifra alarmante que requirió de la toma de acuerdos entre las naciones, en particular, la estimulación de la planificación familiar en los hogares, la capacitación del personal de salud y la organización e implementación de estrategias para la reducción del embarazo en adolescente puesto que en el Ecuador la mayoría de las muertes ocurría entre los 20 y 34 años de edad. ${ }^{23}$

En otra una encuesta sobre demografía y salud sexual se reveló un descenso en los nacimientos, explicando que en la década del 70 del pasado siglo una mujer en edad fértil tenía un promedio de cinco hijos, mientras que con el desarrollo social alcanzado entre el 2007 y el 2012 las mujeres tenían como promedio tres hijos. También se reporta una reducción de la mortalidad infantil en los menores de un año, de manera que por cada 100 nacidos solo 29 fallecieron entre el 1999 y el 2004, en tanto, entre el 2004 y 2012 por cada 100 murieron 13 menores. ${ }^{24}$

Adicionalmente, el informe comenta el incremento de las cesáreas en las mujeres que habitan en áreas rurales a diferencia del comportamiento de la misma variable en área urbana. Siendo las provincias donde más se practica la cesárea Los Ríos, Manabí y El Oro y donde más se estimula y se practica el parto natural en las provincias Zamora Chinchipe, Carchi y Morona Santiago.

Se afirma que cada año nacen niños prematuros y las complicaciones relacionadas con la prematuridad son la principal causa de defunción en los niños menores de 5 años; así en el año 2013 provocaron cerca de un millón de 
muertes y las tres cuartas partes de esas muertes podrían evitarse con intervenciones actuales, eficaces e incluso sin necesidad de recurrir a servicios de cuidados intensivos. ${ }^{25}$

En este sentido, conviene resaltar que, en el Ecuador, la prevalencia de neonatos bajo peso durante los años 1994 a 1999, fue del $16 \%$ en la zona urbana y $19 \%$ en la zona rural. En tanto la tasa de incidencia de bajo peso al nacer se ubicó en $16.1 \%$, es por ello que el bajo peso al nacer constituye en un problema de Salud Pública en el Ecuador. En estudio realizado en el año 2011 en un hospital de la sierra ecuatoriana se determinó una prevalencia de bajo peso al nacer de $41.2 \%$, cifra alarmante que sobrepasa la proyección estimada por el Ecuador según la Organización Mundial de la Salud. ${ }^{26}$ Llama la atención que la atención integral al recién nacido se rige por los protocolos del Ministerio de Salud Pública (MSP), el total de nacimientos en el año 2014 fue de 629 de los cuales 120 fueron neonatos bajo peso, que representa una prevalencia de $19.08 \%$ reconociéndose este como una problemática importante de salud.

Los autores estudiados recogen entre las principales causas de morbilidad en niños las enfermedades gastrointestinales y las diarreicas, las mismas que aportaron cifras a la mortalidad infantil como resultado de la deshidratación. ${ }^{26}$ Siendo estas enfermedades y sus complicaciones altamente prevenible si consideramos el desarrollo científico técnico alcanzado por las ciencias médicas donde las soluciones salinas debidamente dosificadas logran superar los síntomas de la enfermedad y restablecer la salud del niño.

En esta dirección, el estado ecuatoriano, a través del MSP ha diseñado estrategias y programas dirigidos a favorecer la salud de la madre y el niño en especial en la etapa neonatal. Estos programas se han implementado de forma sistemática y gracias a ellos se han modificado algunos indicadores estadísticos de salud, aunque los resultados que hoy se muestran no son los esperados puesto que se requieren de la participación de otros sectores de la sociedad para lograr las metas. Algunos de estos programas gubernamentales que favorecen la salud materno neonatal e infantil son ${ }^{27}$ :

- Norma para el Cuidado Obstétrico y Neonatal Esencial (CONE) en el Sistema Nacional de Salud.

- Ley de Maternidad Gratuita y atención infantil.

- Programa Ampliado de Inmunizaciones.

- Programa de Tamizaje Metabólico Neonatal para detectar Fenilcetonuria, Hipotiroidismo, Insuficiencia suprarrenal y Galactosemia.

- Política de Lactancia materna la misma que ha de ser exclusiva durante los primeros seis meses junto al apego precoz y alojamiento conjunto al momento de nacer.

- Programa de Desnutrición Cero.

- Programa de Salud Escolar bajo el cual se trabaja por las Escuelas saludables y el Programa Nacional de Salud Bucal.

- Plan de Reducción Acelerada de la Muerte Materna y Neonatal.

\section{Conclusiones}

En suma, la morbimortalidad neonatal e infantil es un indicador esencial para evaluar el funcionamiento de los sistemas de salud y al mismo tiempo, de las condiciones de salud y de vida de la población. Este indicador revela la poca eficiencia de la estructura económica social para lograr el crecimiento y desarrollo saludable de los niños.

Los neonatos pueden tener problemas de prematuridad, asfixia, bajo peso e infecciones, mientras que otros, habiendo nacido sanos podrían enfermar con procesos infecciosos o metabólicos en los primeros 28 días, que pondrán en riesgo su vida.

Se hace evidente las inequidades que aún persisten en la sociedad ecuatoriana, en particular, el acceso a servicios de salud, aunque se identificaron pautas para su trasformación se requiere de un programa de seguimiento que ayude a la reducción de las mismas. En este sentido, la atención básica en el nivel primario de atención desempeña un importante papel para la promoción del cuidado a los neonatos de riesgo y a los infantes.

La prevención de la morbimortalidad en neonatos e infantes es el resultado del complejo entramado de determinantes de salud sociales, económicas, culturales y biológicas. Para implementar la prevención, no son suficientes medidas de salud, se deben elaborar políticas y planes de acción integral que minimicen la desigualdad en salud, especialmente entre las mujeres y los neonatos.

En base a lo hasta aquí descrito se recomienda:

Promover el poder real y la capacidad de decisión de fuerzas sociales y comunitarias; resolver las inequidades en las relaciones de poder mediante la reestructuración de los poderes económicos y políticos en la sociedad permitirá influir sobre otros determinantes de la salud y la atención en personas de bajos recursos económicos.

Apoyar a las mujeres en situaciones socioeconómicas pobres y en condiciones de violencia identificadas como vulnerables que tienen mayores riesgos de sufrir complicaciones durante su embarazo que el resto de las mujeres. 
Fomentar el uso de la anticoncepción en adolescentes y mujeres adultas puede ser una medida protectora contra el parto prematuro. El control prenatal con al menos seis visitas al centro de salud de forma planificada y sistemática puede prevenir el parto o nacimiento de niños prematuros u otras complicaciones en la madre y su hijo.

Diseñar intervenciones dirigidas a las mujeres en edad reproductiva que tengan en cuenta sus necesidades en salud sexual y reproductiva, incluyendo uso de anticonceptivos, prevención de embarazos en la adolescencia temprana, prevención de embarazos no deseados, atención prenatal de calidad, cumplimiento del enfoque de riesgo para las gestantes e inclusión de personal calificado en la atención del parto.

\section{Conflicto de intereses}

Ninguno declarado por los autores.

\section{Agradecimientos}

Ninguno declarado por los autores.

\section{Referencias}

1. Organización Mundial de la salud. Reducción de la mortalidad en la niñez. Ginebra. OMS [en línea]. 2016. [citado 2017 Nov 19]. Disponible en: http://www.who.int/mediacentre/factsheets/fs178/es/

2. Unicef. Committing to Child Survival: A Promise Renewed Progress Report 2014. New York; 2014.

3. Brasil. Ministério da Saúde. AIDPI Atenção Integrada às Doenças Prevalentes na Infância: curso de capacitação: introdução: módulo 1. 2. edição rev. 1.a reimpressão. Brasília: Editora Ministério da Saúde, 2003. 32p.

4. Domínguez LP, Torres PG, Díaz JC, Gutiérrez MV. Prematuro tardío: morbilidad y mortalidad en la Unidad de Cuidados Intensivos Neonatales. Revista Médica Hospital General de México. 2013: 29-33.

5. Espelt, M., Frezza, L., Ratchi, L., Sánchez, G., \& Meritano, J. (2012). Morbilidad Asociada a Recién Nacidos Pre término Tardío. Revista Hospital Materno Infantil Ramón Sardi.

6. Bajaña Chávez, GA.Determinación de morbimortalidad de neonatos pretérmino nacidos en el Hospital Abel Gílbert Pontón en el año 2013. [Tesis de grado]. Universidad Católica Santiago de Guayaquil. Ecuador; 2013.

7. Instituto Nacional de Estadísticas y Censos. Anuario de Estadísticas Vitales - Nacimientos y Defunciones 2014. Quito; 2014.

8. Conde A, Diaz JL, Belizan JM. Método madre canguro para reducir la morbimortalidad en neonatos con bajo peso al nacer. Biblioteca CochranePlus [en línea]. 2008. [citado 2017 Nov 19]. Disponible en: http://cochrane.bvsalud.org/cochrane/main.php?lib=BCP\&searchExp=método

9. Campa M. Algunos factores de riesgo de la mortalidad infantil, en un área de salud. [en línea]. s/f. [citado 2017 Nov 19]. Disponible en: http://www.scielo.org//cgibin/wxis.exe/applications/scieloorg/iah/?lang=p\&lsisScript=iah/iah.xis\&base=article^dart.org\&nextAction=search\&exprSearch=muert e\%20materna\&form $=$

10. Ecuador. Ministerio de Salud Pública. Hospital Gineco Obstétrico Isidro Ayora. Planificación estratégica: 2015-2017. Quito; 2015.

11. Orbe Galárraga EG, Vinelli Merino W. Morbimortalidad en neonatos hospitalizados en Cuidados Intensivos Neonatales Hospital Gineco - Obstétrico Isidro Ayora Quito octubre 2014 - octubre 2016. [Tesis de posgrado]. Universidad Central del Ecuador. Ecuador; 2016.

12. Brasil. Ministério da Saúde. Agenda de compromissos para a saúde integral da criança e redução da mortalidade infantil. 1a edição. Brasília: Editora do Ministério da Saúde, 2004. 80 p.

13. WHO. Born Too Soon: The Global Action Report on Preterm Birth. Eds. Howson CP, Kinney MV, Lawn JE. World Health Organization. Geneva; 2012.

14. Araujo BF, Madi JM, Coelho MB, Zatti H, Olmi FB, Canabarro CT. Analysis of neonatal morbidity and mortality in late preterm newborn infants. Journal de Pediatría. 2012; 88.

15. Rodríguez JR, Martínez YC, Rodríguez DZ. Factores de riesgo relacionados con bajo peso al nacer en el municipio Cienfuegos. Medisur. 2015;13(3).

16. PeriStats. White Plains, NY: March of Dimes[en línea]. 2006. [citado 2017 Nov 19]. Disponible en: http://www.marchofdimes.com/peristats/

17. López Losano ME. Problemática de la salud neonatal, factores asociados y estrategias para afrontar en el Perú. Ciencias de la Salud. [en línea]. 2015. [citado 2017 Nov 19]; 2(1): 402-410. Disponible en: http://revistas.uladech.edu.pe/index.php/increscendo-salud/article/view833//531

18. Ipiales Villegas SA, Carrera Domínguez NB. Morbilidad y mortalidad del recién nacido bajo peso en el Hospital IESS en Ambato[en línea]. 2014. [citado 2017 Nov 19]. Disponible en: http://dspace.uniandes.edu.ec/handle/123456789/3693

19. Brasil. Ministério da Saúde. Agenda de compromissos para a saúde integral da criança e redução da mortalidade infantil. 1a edição. Brasília: Editora do Ministério da Saúde; 2004.

20. Wilson KA, Wallace DD, Goudar SS, Theriaque D, McClure EM. Identifying Causes of Neonatal Mortality from Observational Data: A Bayesian Network Approach. In Proceedings of the International Conference on Data Mining (DMIN) (p. 132). The Steering Committee of The World Congress in Computer Science, Computer Engineering and Applied Computing (WorldComp); 2015.

21. Organización Mundial de la Salud. Declaración de Alma Ata; 1978.

22. Laspina Arellano N, Villota Villota L, Castillo M. Impacto de las intervenciones de salud sobre la mortalidad infantil en Ecuador, período 1960-2013. Rev Fac Cien Med (Quito). 2016; 41(1): 57-70.

23. Mendoza LA, Arias M, Mendoza LI. Hijo de madre adolescente: riesgos, morbilidad y mortalidad neonatal. Rev. chil. obstet. ginecol. [en línea]. 2012 [citado 2017 Nov 19]; 77( 5 ): 375-382. Disponible en: http://www.scielo.cl/scielo.php?script=sci arttext\&pid=S071775262012000500008\&Ing=es

24. INEC. Censo de población y vivienda 2014. anuario de estadísticas vitales: nacimientos y defunciones 2014. [en línea]. 2014. [citado 2017 Nov 19]. Disponible en: http://www.ecuadorencifras.gob.ec/nacimientos-defunciones

25. Organización mundial de la salud. Nacimientos prematuros. Ginebra. OMS [en línea]. 2015. [citado 2017 Nov 19]. Disponible en: http://www.who.int/mediacentre/factsheets/fs363/es/

26. Villegas Ipiales SA, Cabrera Domínguez NB. Morbilidad y mortalidad del recién nacido bajo peso en el hospital IESS Ambato. Año 2014. [Tesis de grado]. UNIANDES, Ecuador; 2016.

27. Ministerio de Salud Pública del Ecuador. Ministerio de Salud Pública, Dirección Nacional de Normatización-MSP; 2013. 